\title{
Structure of the Electron Distribution Function and Induced Beam Instability in Collisionless Magnetic Reconnection with a Strong Guide Field
}

\author{
Kazuya SHIMOMURA, Tomohiko WATANABE, Shinya MAEYAMA and Akihiro ISHIZAWA ${ }^{1)}$ \\ Department of Physics, Nagoya University, Nagoya 464-8602, Japan \\ ${ }^{1)}$ Graduate School of Energy Science, Kyoto University, Uji 611-0011, Japan
}

(Received 15 June 2020 / Accepted 25 September 2020)

\begin{abstract}
A phase space structure of the electron distribution function is investigated by the gyrokinetic theory and numerical simulations to investigate a possible mechanism of the excitation of the beam instability which induces collisionless magnetic reconnection in a strong guide field. It is shown that the perturbed electron distribution function develops in proportion to the shifted Maxwellian distribution as the reconnection electric field accelerates electrons along the guide field at the X-point, with parity symmetry around the $z$ - axis. The accelerated electrons are expected to excite the kinetic Alfvén waves (KAWs) when the beam velocity exceeds the Alfvén speed. The obtained results suggest a possible scenario for anomalous resistivity generation in the case with the strong guide field where the beam electrons accelerated at the X-point lose their parallel momentum through interactions with the self-excited KAWs.

(C) 2020 The Japan Society of Plasma Science and Nuclear Fusion Research
\end{abstract}

Keywords: magnetic reconnection, gyrokinetic simulation, kinetic Alfvén wave, beam instability, anomalous instability, collisionless reconnection

DOI: $10.1585 /$ pfr. 15.1401084

\section{Introduction}

Magnetic reconnection refers to the topological change of magnetic field lines; it is a fundamental physical process that induces the conversion of magnetic energy to kinetic energy, leading to the self-organization of plasmas in the laboratory and space. Simulation studies on magnetic reconnection have been conducted based on the magnetohydrodynamics (MHD) model [1,2], where resistivity plays a role in the dissipation process at the reconnection point $(\mathrm{X})$. However, as is well known, the Spitzer resistivity is overly small to facilitate the fast reconnection events in space and fusion plasmas, such as the sawtooth crash. Therefore, two-fluid effects or the kinetic effects are considered important for realizing sufficiently fast magnetic reconnection [3-12].

Anomalous resistivity is one of the factors that continuously drive the magnetic reconnection in collisionless plasmas. Over the years, the anomalous resistivity has been discussed regarding its induction during the magnetic reconnection and how it facilitates a fast reconnection. Conventionally, the lower hybrid drift instability and the drift kink instability can induce the anomalous resistivity in collisionless magnetic reconnection without a guide field $[13,14]$. In magnetic reconnection with a strong magnetic guide field, e.g., the sawtooth crash, the particle orbit effects related to the nongyrotropic motion may be subsidiary. Conversely, the anomalous resistivity or the elec- tron inertia effect may have a significant impact on the collisionless reconnection with the strong guide field. Recent measurements by the magnetospheric multiscale science mission have shown that the reconnection rate is higher than that predicted by the Hall- MHD model, implying that the anomalous resistivity enhances the reconnection rate [15]. Furthermore, in a recent simulation study [16], it was shown that the anomalous resistivity driven by the Buneman instability accelerates the reconnection rate. As is known, the Buneman instability is driven by super thermal electrons; however, the mechanism through which the unstable condition is realized at the X-point is unclear.

In strongly magnetized plasmas, the collective motions of charged particles can be described using the gyrokinetic equations in a characteristic time scale longer than the gyro period [17]. The gyrokinetic equations are often used for the analysis of turbulent transport in fusion plasmas [18], and they were recently applied to the magnetic reconnection with a strong guide field [19-21].

We postulate the beam instability of the kinetic Alfvén waves (KAWs) as a possible cause of the anomalous resistivity in the collisionless reconnection with a strong guide field, and discuss the spontaneous destabilization process with the structural changes in electromagnetic fields and the distribution function during the magnetic reconnection. Our arguments are twofold. First, an analytic solution of the collisionless gyrokinetic equations for the distribution function at the X-point is derived using the symmetry of 
the system under the gyrokinetic ordering. It is revealed that the deviation of the gyrocenter distribution function $\tilde{f}_{\| s}$ from the initial condition is proportional to $v_{\|} F_{M}$ at the $\mathrm{X}$-point, where $v_{\|}$is the velocity along the magnetic field and $F_{M}$ is the Maxwellian distribution. To our knowledge, no analytic solution of the velocity distribution function at the X-point has been reported in the literature, although the electron acceleration was expected from the extended MHD or two-fluid models. Second, the analytic solution of $\tilde{f}_{\| s}$ exhibits a beam velocity proportional to the reconnected flux. Thus, as the magnetic reconnection develops, the beam velocity may exceed the Alfvén speed, leading to the destabilization of the KAWs. This suggests that the unstable condition of KAWs may be spontaneously satisfied during the collisionless magnetic reconnection, which provides us with a possible scenario of the self-generation of anomalous resistivity.

A conventional kinetic simulation study was conducted on the Buneman instability with a parallel wavelength of the order of the ion skin depth, $d_{i}[16]$; however, the researchers were unable to investigate the KAWs with much longer parallel wavelengths, $k_{\|} \ll d_{i}^{-1}$. Conversely, the present study focuses on the latter using the gyrokinetic theory with $k_{\|} / k_{\perp} \ll 1$. Additionally, considering a finite beta plasma, $\beta_{e} \gg m_{e} / m_{i}$, with respect to the electron-to-ion mass ratio, the thermal electron speed is established to be much larger than the Alfvén one. Thus, the electron beam developed via magnetic reconnection may satisfy the instability condition of KAWs, which is easily fulfilled compared with the Buneman instability condition. Therefore, the spontaneous realization of the Buneman instability condition is difficult during the collisionless reconnection. In this work, we conducted 2-D gyrokinetic simulations to elucidate the generation of the beam distribution function under the collisionless magnetic reconnection, and the stability of the KAWs under a simplified beam distribution. The unified simulation of the magnetic reconnection and the self-consistent beam instability induced during the reconnection will be considered in a future study.

The present study is organized as follows: in Sec. 2, we describe a gyrokinetic model for the collisionless reconnection and discuss structures of the distribution function at the X-point from a viewpoint of symmetry in the system. In Sec. 3, we discuss the beam instability of the KAWs using the linear dispersion relation. In Sec. 4, the formation of the electron beam is elucidated by gyrokinetic simulations of the collisionless reconnection. Summary and discussions are provided in Sec. 5 .

\section{Model}

\subsection{Gyrokinetic equations}

We consider the same gyrokinetic model in a slab plasma as that in Ref. [20], where the uniform guide field is given by $\boldsymbol{B}_{0}=B_{0} \hat{z}$, with the translational symmetry in the $z$ direction. The distribution function is divided into equilibrium and perturbed parts, $\left(F_{\text {tots }}=F_{M s}+\tilde{f}_{s}\right)$, where $F_{\text {tots }}$ and $F_{M s}$ represent the total and the Maxwellian distribution functions, respectively. The subscript, $s$, denotes the particle species ( $s=i$ for ions and $s=e$ for electrons). We assume the delta-f gyrokinetic ordering, $\epsilon_{g k} \sim \tilde{f}_{s} / F_{M s} \sim \tilde{v}_{E \times B} / v_{t i} \sim \tilde{B}_{\perp} / B_{0} \sim \rho_{i} / L \ll 1$, where $\tilde{v}_{E \times B}, v_{t i}, \tilde{B}_{\perp}, \rho_{s}$, and $L$ are the $\boldsymbol{E} \times \boldsymbol{B}$ drift velocity, the thermal speed, the perpendicular component of perturbed magnetic field, the thermal gyroradius, and a characteristic scale, respectively. Throughout the present study, the symbol, , represents the perturbed quantities of the order of $O\left(\epsilon_{g k}\right)$. For simplicity, it is also assumed that $\tilde{f}_{s}$ has the Maxwellian distribution in the perpendicular velocity space, i.e., $\tilde{f}_{s k}\left(v_{\|}, v_{\perp}\right)=\tilde{f}_{\| s k}\left(v_{\|}\right) F_{M s \perp}\left(v_{\perp}\right)$.

The time evolution of the perturbed distribution function, $\tilde{f}_{\| s k}$, in the perpendicular wavenumber space $(\boldsymbol{k})$ is described by the gyrokinetic Vlasov equation integrated over $v_{\perp}$, the quasi-neutrality conditions, and the Ampére law:

$$
\begin{aligned}
& \partial_{t} \tilde{f}_{\| s \boldsymbol{k}}-\frac{e_{s}}{T_{s}} v_{\|} F_{M \| s} \tilde{E}_{\| s \boldsymbol{k}} \\
& -\sum_{\boldsymbol{k}^{\prime}} \sum_{\boldsymbol{k}^{\prime \prime}} \delta_{\boldsymbol{k}^{\prime}+\boldsymbol{k}^{\prime \prime}, \boldsymbol{k}} \frac{\hat{\boldsymbol{z}} \cdot\left(\boldsymbol{k}^{\prime} \times \boldsymbol{k}^{\prime \prime}\right)}{B_{0}} J_{0 s k^{\prime}}\left(\tilde{\phi}_{\boldsymbol{k}^{\prime}}-v_{\|} \tilde{A}_{\| \boldsymbol{k}^{\prime}}\right) \tilde{f}_{\| s \boldsymbol{k}^{\prime \prime}} \\
& =0 \\
& \sum_{s} e_{s} \int d v_{\|} J_{0 s k} \tilde{f}_{\| s \boldsymbol{s}}=\sum_{s} \frac{e_{s}^{2} n_{0}}{T_{s}}\left(1-\Gamma_{0 s k}\right) \tilde{\phi}_{\boldsymbol{k}} \\
& k_{\perp}^{2} \tilde{A}_{\| \boldsymbol{k}}=\mu_{0} \sum_{s} e_{s} \int d v_{\|} v_{\|} J_{0 s k} \tilde{f}_{\| s \boldsymbol{s}}
\end{aligned}
$$

where

$$
\begin{aligned}
\tilde{E}_{\| s \boldsymbol{k}}= & -\partial_{t} J_{0 s k} \tilde{A}_{\| \boldsymbol{k}} \\
& +\sum_{\boldsymbol{k}^{\prime}} \sum_{\boldsymbol{k}^{\prime \prime}} \delta_{\boldsymbol{k}^{\prime}+\boldsymbol{k}^{\prime \prime}, \boldsymbol{k}} \frac{\hat{\boldsymbol{z}} \cdot\left(\boldsymbol{k}^{\prime} \times \boldsymbol{k}^{\prime \prime}\right)}{B_{0}} J_{0 s k^{\prime}} \tilde{\phi}_{\boldsymbol{k}^{\prime}} J_{0 s k^{\prime \prime}} \tilde{A}_{\| \boldsymbol{k}^{\prime \prime}},
\end{aligned}
$$

$\Gamma_{0 s k}=I_{0}\left(b_{s k}\right) e^{-b_{s k}}$ with $b_{s k}=k_{\perp}^{2} \rho_{s}^{2}$, the modified Bessel function $I_{0}$ and $J_{0 s k}=\exp \left(-b_{s k} / 2\right) . \tilde{E}_{\|}, \tilde{\phi}, \tilde{A_{\|}}, e_{s}, T_{s}$, and $n_{0}$ are the parallel electric field, perturbed electrostatic potential, parallel component of perturbed vector potential, electric charge, temperature, and equilibrium number density.

The gyrokinetic equations, Eqs. (1) - (3), are rewritten in the real space

$$
\begin{aligned}
& \partial_{t} \tilde{f}_{\| s}-\frac{e_{s}}{T_{s}} v_{\|} F_{M \| s}\left\langle\tilde{E}_{\|}\right\rangle_{s}+\left[\left\langle\tilde{\phi}-v_{\|} \tilde{A}_{\|}\right\rangle_{s}, \tilde{f}_{\| s}\right]=0, \\
& \sum_{s} e_{s} \int d v_{\|}\left\langle\tilde{f}_{\| s}\left(\boldsymbol{x}, v_{\|}, t\right)\right\rangle_{s} \\
& \quad=\sum_{s} \frac{e_{s}^{2} n_{0}}{T_{s}}\left(\tilde{\phi}(\boldsymbol{x}, t)-\langle\langle\tilde{\phi}(\boldsymbol{x}, t)\rangle\rangle_{s}\right), \\
& \nabla_{\perp}^{2} \tilde{A}_{\|}(\boldsymbol{x}, t)=-\mu_{0} \sum_{s} e_{s} \int d v_{\|} v_{\|}\left\langle\tilde{f}_{\| s}\left(\boldsymbol{x}, v_{\|}, t\right)\right\rangle_{s}
\end{aligned}
$$

where the gyrophase-average operators for an arbitrary function, $\tilde{g}(\boldsymbol{x})$, are defined as $\langle\tilde{g}(\boldsymbol{x})\rangle_{s}=\left(1 / L_{x} L_{y}\right)$ $\int d \boldsymbol{x}^{\prime} J_{0 s}\left(\boldsymbol{x}^{\prime}\right) \tilde{g}\left(\boldsymbol{x}-\boldsymbol{x}^{\prime}\right)$ and $\langle\langle\tilde{g}(\boldsymbol{x})\rangle\rangle_{s}=\left(1 / L_{x} L_{y}\right)$ 
$\int d \boldsymbol{x}^{\prime} \Gamma_{0 s}\left(\boldsymbol{x}^{\prime}\right) \tilde{g}\left(\boldsymbol{x}-\boldsymbol{x}^{\prime}\right)$, with the gyroradius given as $\rho_{s}=$ $m_{s} v_{t s} / e_{s} B_{0}$, the particle mass $m_{s}$, and the thermal speed $v_{t s}=\sqrt{T_{s} / m_{s}} \cdot J_{0 s}(\boldsymbol{x}) \equiv \sum_{k} \exp (i \boldsymbol{k} \cdot \boldsymbol{x}) J_{0 s k}$ and $\Gamma_{0 s}(\boldsymbol{x}) \equiv$ $\sum_{k} \exp (i \boldsymbol{k} \cdot \boldsymbol{x}) \Gamma_{0 s k}$. The perturbed distribution function, $\tilde{f}_{\| s}\left(\boldsymbol{x}, v_{\|}, t\right)$, is a function of the gyrocenter position $(\boldsymbol{x}) . L_{x}$ and $L_{y}$ refer to the system size in the $x$ and $y$ directions with the periodic boundary condition, respectively. The nonlinear term in the gyrokinetic Vlasov equation, Eq. (4), is represented in terms of the Poisson's brackets showing the $E \times B$ drift and the advection along the perturbed magnetic field. The Poisson brackets in the gyrocenter coordinates are denoted by $[\tilde{g}, \tilde{h}]$ where $[\tilde{g}, \tilde{h}]=\left(\partial_{x} \tilde{g} \partial_{y} \tilde{h}-\partial_{y} \tilde{g} \partial_{x} \tilde{h}\right) / B_{0}$. The parallel electric field is given by

$$
\left\langle\tilde{E}_{\|}\right\rangle_{s}=-\partial_{t}\left\langle\tilde{A}_{\|}\right\rangle_{s}-\left[\langle\tilde{\phi}\rangle_{s},\left\langle\tilde{A}_{\|}\right\rangle_{s}\right]
$$

\subsection{Symmetry of the system}

In the collisionless gyrokinetic system for the magnetic reconnection, we verify the preservation of the parity symmetry which is utilized to derive the analytic solution of the gyrocenter distribution function at the $\mathrm{X}$-point. Here, we consider the initial condition:

$$
\begin{aligned}
& \tilde{f}_{\| s}\left(x, y, v_{\|}, t=0\right) \\
& =\frac{\tilde{n}_{\text {init }}}{\sqrt{2 \pi} v_{t s}} \exp \left[-\frac{\left(v_{\|}-U_{0 s} \cos \left(\frac{2 \pi x}{L_{x}}\right)\right)^{2}}{2 v_{t s}^{2}}\right] \\
& \quad+\epsilon \cos \left(\frac{2 \pi x}{L_{x}}\right) \cos \left(\frac{2 \pi y}{L_{y}}\right) F_{M \| s .} .
\end{aligned}
$$

The first term on the right indicates the shifted Maxwellian distribution, which produces the initial profile of $\tilde{A}_{\|}$in proportion to $\tilde{n}_{\text {init }} U_{0 s} \cos \left(\frac{2 \pi x}{L_{x}}\right)$. The second term is a small seed that triggers the reconnection. The reconnecting field is included in the perturbed part, not in the background part. Notably, the parallel drift velocity, $U_{0 s}$, can be $O\left(v_{t s}\right)$, whereas the amplitude providing the perturbed number density, $\tilde{n}_{\text {init }}$, is $O\left(\epsilon_{g k} n_{0}\right)$. Therefore, the perturbed parallel flow given by $\tilde{f}_{\| s}$ is $O\left(\epsilon_{g k} v_{t s}\right)$, which is consistent with the conventional delta-f gyrokinetic ordering.

The initial condition has a symmetry for the reversal of the coordinates around the X-point, that is, i.e., $\tilde{f}_{\| s}\left(x, y, v_{\|}, t=0\right)=\tilde{f} \| s_{\| s}\left(-x,-y, v_{\|}, t=0\right)$. Equations (4) (6) are invariant under the coordinate transformation of $(x, y) \rightarrow(-x,-y)$. We divide $\tilde{f}_{\| s}$ into even parity $\left(\tilde{f}_{s^{+}}\right)$and the odd parity $\left(\tilde{f}_{s^{-}}\right)$components:

$$
\begin{aligned}
& \tilde{f}_{s+}\left(\boldsymbol{x}, v_{\|}\right)=\frac{\tilde{f}_{\| s}\left(\boldsymbol{x}, v_{\|}\right)+\tilde{f}_{\| s}\left(-\boldsymbol{x}, v_{\|}\right)}{2}, \\
& \tilde{f}_{s-}\left(\boldsymbol{x}, v_{\|}\right)=\frac{\tilde{f}_{\| s}\left(\boldsymbol{x}, v_{\|}\right)-\tilde{f}_{\| s}\left(-\boldsymbol{x}, v_{\|}\right)}{2},
\end{aligned}
$$

so that $\tilde{f}_{s+}\left(\boldsymbol{x}, v_{\|}, t\right)=\tilde{f}_{s^{+}}\left(-\boldsymbol{x}, v_{\|}, t\right)$ and $\tilde{f}_{s_{-}}\left(\boldsymbol{x}, v_{\|}, t\right)=$ $-\tilde{f}_{s-}\left(-\boldsymbol{x}, v_{\|}, t\right)$. Similarly, $\tilde{E}_{\| s+}, \tilde{E}_{\| s-}, \tilde{\phi}_{+}, \tilde{\phi}_{-}, \tilde{A}_{\|+}$and $\tilde{A}_{\|-}$ are defined. Thus, Eq. (4) is rewritten as

$$
\partial_{t} \tilde{f}_{s+}=v_{\|} \frac{e_{s}}{T_{s}} F_{M \| s}\left\langle\tilde{E}_{\|}\right\rangle_{s+}-\left[\left\langle\tilde{\phi}_{+}-v_{\|} \tilde{A}_{\|+}\right\rangle_{s}, \tilde{f}_{s+}\right]
$$

$$
\begin{aligned}
& -\left[\left\langle\tilde{\phi}_{-}-v_{\|} \tilde{A}_{\|-}\right\rangle_{s}, \tilde{f}_{s-}\right], \\
\partial_{t} \tilde{f}_{s^{-}}= & v_{\|} \frac{e_{s}}{T_{s}} F_{M \| s}\left\langle\tilde{E}_{\|}\right\rangle_{s-}-\left[\left\langle\tilde{\phi}_{+}-v_{\|} \tilde{A}_{\|+}\right\rangle_{s}, \tilde{f}_{s-}\right] \\
& -\left[\left\langle\tilde{\phi}_{-}-v_{\|} \tilde{A}_{\|-}\right\rangle_{s}, \tilde{f}_{s+}\right] .
\end{aligned}
$$

If the initial condition of $\tilde{f}_{\| s}\left(\boldsymbol{x}, v_{\|}, t=0\right)$ has the even parity only, like Eq. (8) (namely $\tilde{f}_{s^{-}}=0$ ), Eqs. (11) and (12) become

$$
\begin{aligned}
& \partial_{t} \tilde{f}_{s+}=v_{\|} \frac{e_{s}}{T_{s}} F_{M \| s}\left\langle\tilde{E}_{\|}\right\rangle_{s+}-\left[\left\langle\tilde{\phi}_{+}-v_{\|} \tilde{A}_{\|+}\right\rangle_{s}, \tilde{f}_{s+}\right], \\
& \partial_{t} \tilde{f}_{s-}=0 .
\end{aligned}
$$

Thus, the perturbed distribution function preserves the parity symmetry around the X-point,

$$
\tilde{f}_{\| s}\left(\boldsymbol{x}, v_{\|}, t\right)=\tilde{f}_{\| s}\left(-\boldsymbol{x}, v_{\|}, t\right)
$$

Substituting $\tilde{f}_{s+}$ for $\tilde{f}_{\| s}$ in Eqs. (5) and (6), $\langle\tilde{\phi}\rangle_{s}$ and $\left\langle\tilde{A}_{\|}\right\rangle_{s}$ also preserves the parity symmetry around the $\mathrm{X}$-point,

$$
\begin{aligned}
& \langle\tilde{\phi}\rangle_{s}(\boldsymbol{x}, t)=\langle\tilde{\phi}\rangle_{s}(-\boldsymbol{x}, t), \\
& \left\langle\tilde{A}_{\|}\right\rangle_{s}(\boldsymbol{x}, t)=\left\langle\tilde{A}_{\|}\right\rangle_{s}(-\boldsymbol{x}, t) .
\end{aligned}
$$

Here, the "even" and "odd" parities are defined in terms of the symmetry with respect to the $\mathrm{X}$ point (not the $y$ axis), where Eqs. (16) and (17), respectively, show the signs of $\phi$ and $A_{\|}$unchanged for the rotation of $\pm \pi$, not for the reversal of either $x$ or $y$. Thus, $\phi$ and $A_{\|}$possess the odd parity with respect to the reversal of $x$, consistent with the tearing mode theory. Noteworthily, the even parity mode considered here contains an "asymmetric" profile with respect to the $y$ axis.

\subsection{Derivation of the distribution functions at the $X$-point}

On the basis of the above discussion, we find that the Poisson's brackets vanish at the X-point $(\boldsymbol{x}=0)$ where $\partial_{x}=0$ and $\partial_{y}=0$, if $\tilde{f}_{\| s}$ as well as $\langle\tilde{\phi}\rangle_{s}$ and $\left\langle\tilde{A}_{\|}\right\rangle_{s}$ is symmetric in Eqs. (15) - (17). Hence, Eq. (4) is reduced to

$$
\partial_{t} \tilde{f}_{\| s}+\frac{e_{s}}{T_{s}} v_{\|} F_{M \| s} \partial_{t}\left\langle\tilde{A}_{\|}\right\rangle_{s}=0
$$

at the X-point. This implies that the structure of the distribution function at the $\mathrm{X}$-point is determined only by the parallel acceleration because of the induction electric field. In other words, the reconnection electric field $\left\langle\tilde{E}_{\|}\right\rangle_{s}$ is balanced with the inertia force; thus, the inertia is a cause of the collisionless reconnection in the present model. We find the solution of $\tilde{f}_{\| s}$ at the X-point by integrating Eq. (18) over $t$ :

$$
\begin{aligned}
& \tilde{f}_{\| s}\left(\boldsymbol{x}=0, v_{\|}, t\right)-\tilde{f}_{\| s}\left(\boldsymbol{x}=0, v_{\|}, t=0\right) \\
& =-\frac{e_{s}}{T_{s}}\left(\left\langle\tilde{A}_{\|}\right\rangle_{s}(\boldsymbol{x}=0, t)-\left\langle\tilde{A}_{\|}\right\rangle_{s}(\boldsymbol{x}=0, t=0)\right) v_{\|} F_{M \| s} .
\end{aligned}
$$


The time variation of the perturbed distribution function $\tilde{f}_{\| s}$ at the X-point is proportional to the reconnected magnetic flux:

$$
\Delta \tilde{A}_{\| s} \equiv-\frac{e_{s}}{m_{s}}\left(\left\langle\tilde{A}_{\|}\right\rangle_{s}(\boldsymbol{x}=0, t)-\left\langle\tilde{A}_{\|}\right\rangle_{s}(\boldsymbol{x}=0, t=0)\right),
$$

and it has a velocity dependence of the shifted Maxwellian $v_{\|} F_{M \| s}$. This means that the induced electric field at the $\mathrm{X}$-point generates electron and ion beams. The generated beam velocity, $\Delta \tilde{U}_{s}$, is evaluated at the X-point using the gyrocenter distribution function:

$$
\begin{aligned}
& \Delta \tilde{U}_{s}(\boldsymbol{x}=0, t)=\tilde{U}_{s}(t)-\tilde{U}_{s}(0) \\
& \quad=\frac{1}{n_{0}} \int d v_{\|} v_{\|} \tilde{f}_{\| s}\left(\boldsymbol{x}=0, v_{\|}, t\right)-\frac{\tilde{n}_{\text {init }}}{n_{0}} U_{0 s},
\end{aligned}
$$

where $\tilde{U}_{s}=1 / n_{0} \int d v_{\|} v_{\|} \tilde{f}_{\| s}\left(\boldsymbol{x}=0, v_{\|}, t\right)$, and $U_{0 s}$ is the initial averaged velocity at the X-point (see Eq. (8)). From Eqs. (19) - (21), $\Delta \tilde{U}_{s}=\Delta \tilde{A}_{\| s} \sim \epsilon_{g k} v_{t s}$.

Here, we discuss the energy conversion from the magnetic energy, $\int d x d y \tilde{B}_{\perp}^{2} / 2$, to the parallel electron beam energy, $\int d x d y m_{e} n_{0} \tilde{U}_{e}^{2} / 2$,

$$
\int_{S_{d r}} d x d y \frac{m_{e} n_{0} \tilde{U}_{e}^{2}}{2}=\alpha \int_{S_{s i m u}} d x d y \frac{\tilde{B}_{\perp}^{2}}{2 \mu_{0}},
$$

where $\alpha$ is the energy conversion rate of the electron kinetic energy accelerated along the guide field in the small diffusion region to the magnetic energy released during the magnetic reconnection in the region characterized by the ion gyroradius scale and $\tilde{B}_{\perp} \sim \epsilon_{g k} B_{0}$. Assuming that a part of the magnetic energy released in the simulation region, $S_{\text {simu }}$, is converted to the beam energy in the diffusion region, $S_{d r}$, we find that

$$
\tilde{U}_{e}^{2}=\alpha \epsilon_{g k}^{2} \frac{m_{i}}{m_{e}} V_{A}^{2} \frac{S_{\text {simu }}}{S_{d r}},
$$

with an Alfvén speed of $V_{A}^{2}=B_{0}^{2} / \mu_{0} m_{i} n_{0}$. It is roughly estimated that $S_{\text {simu }} \sim L_{x}^{2}>\rho_{i}^{2}$ and $S_{d r} \sim d_{e}^{2}$ where $L_{x}$ is the simulation box size and $d_{e}$ is the electron skin depth. Thus, Eq. (23) is reduced to

$$
\tilde{U}_{e}>\sqrt{\alpha \frac{m_{i}}{m_{e}}} \frac{\epsilon_{g k}}{\epsilon_{a}} V_{A}=\sqrt{\alpha \frac{m_{i}}{m_{e}}} \epsilon_{g k} v_{t e},
$$

where $V_{A}=\epsilon_{a} v_{t e}$ and $\epsilon_{a}=\left(m_{e} / m_{i} \beta_{i}\right)^{1 / 2}$. Thus, from the viewpoint of energy balance, we find that $\tilde{U}_{e} \sim \epsilon_{g k} v_{t e}$, consistent with the delta-f ordering, whereas the super Alfvénic beam velocity, $\tilde{U}_{e}>V_{A}$ can be generated in the case of $\sqrt{\alpha m_{i} / m_{e}} \sim 1$ and $\epsilon_{g k}>\epsilon_{a}$.

\subsection{Comparison with MHD and Vlasov models}

The comparison results between the gyrokinetic model and a reduced MHD model are discussed below. Assuming the long wavelength approximation of $k_{\perp} \rho_{i} \ll 1$, we neglect the higher order terms of $k_{\perp}$ by expanding the gyrophase average operators. The approximated forms are $J_{0 s k} \sim 1$ and $\Gamma_{0 e k} \sim 1$, whereas the lowest order term of the ion polarization is maintained, i.e., $\Gamma_{0 i k} \sim 1-k_{\perp}^{2} \rho_{i}^{2}$. The reduced gyrophase average operators are summarized as follows:

$$
\begin{aligned}
& J_{0 s}(\boldsymbol{x}) \sim 1, \\
& \Gamma_{0 s} \sim \begin{cases}1 & (\text { for } s=e) \\
1+\rho_{i}^{2} \nabla_{\perp}^{2} & (\text { for } s=i) .\end{cases}
\end{aligned}
$$

Taking $v_{\|}$integrals of Eq. (4) multiplied by $e_{s}$ and $e_{s} a_{s} v_{\|}$ and summmed over $s$, individually, the zeroth and the first order moments of the gyrokinetic Vlasov equation are derived using Eqs. (5) and (6):

$$
\begin{aligned}
& \partial_{t} \nabla_{\perp}^{2} \tilde{\phi}+\left[\tilde{\phi}, \nabla_{\perp}^{2} \tilde{\phi}\right]=V_{A}^{2}\left[\tilde{J}_{\|}, \tilde{A}_{\|}\right] \\
& \partial_{t}\left(\sum_{s} \frac{e_{s}^{2} n_{0}}{m_{s}} \tilde{A}_{\|}+\frac{\tilde{J}_{\|}}{\mu_{0}}\right)+\left[\tilde{\phi}, \sum_{s} \frac{e_{s}^{2} n_{0}}{m_{s}} \tilde{A}_{\|}+\frac{\tilde{J}_{\|}}{\mu_{0}}\right] \\
& \quad-\left[\tilde{A}_{\|}, \sum_{s} \int d v_{\|} e_{s} v_{\|}^{2} \tilde{f}_{\| s}\right]=0
\end{aligned}
$$

where $\tilde{J}_{\|}=-\nabla_{\perp}^{2} \tilde{A}_{\|}$. When the moment hierarchy is truncated at the second order in the low- $\beta$ limit and the ion parallel current is neglected, Eq. (28) is rewritten as

$$
\partial_{t} \tilde{F}+[\tilde{\phi}, \tilde{F}]=0
$$

where $\tilde{F}=\tilde{J}_{\|}+d_{e}^{2} \tilde{A}_{\|}$. Therefore, in this limit, Eqs. (27) and (29) are equivalent to the vorticity equation and the Ohm's law in the imcompressible 2-D slab model of the extended MHD equations [5], respectively. Thus, the same argument on the parity symmetry around the X-point can be applied to the extended MHD equations. However, the validity of the long wavelength approximation is questionable around the X-point, where structures with $k_{\perp} \rho_{i}>1$ often develop in the nonlinear phase. Therefore, the analyses based on the gyrokinetic equations or the Vlasov-Maxwell equations are essential toward the quantitative understanding of the collisionless reconnection.

Here, we discuss the symmetry property around the $\mathrm{X}$-point using the collisionless Vlasov-Maxwell equations in the five dimensions of phase space:

$$
\begin{aligned}
& \partial_{t} f_{s}\left(\boldsymbol{x}_{\perp}, \boldsymbol{v}_{\perp}, v_{z}\right)+\boldsymbol{v} \cdot \nabla f_{s}\left(\boldsymbol{x}_{\perp}, \boldsymbol{v}_{\perp}, v_{z}\right) \\
& \quad+\frac{e_{s}}{m_{s}}\left(\boldsymbol{E}\left(\boldsymbol{x}_{\perp}\right)+\boldsymbol{v} \times \boldsymbol{B}\left(\boldsymbol{x}_{\perp}\right)\right) \cdot \partial_{\boldsymbol{v}} f_{s}\left(\boldsymbol{x}_{\perp}, \boldsymbol{v}_{\perp}, v_{z}\right)=0 \\
& \partial_{t} \boldsymbol{B}=-\nabla \times \boldsymbol{E} \\
& \frac{1}{\epsilon_{0}} \partial_{t} \boldsymbol{E}=\nabla \times \boldsymbol{B}-\mu_{0} \boldsymbol{J} \\
& \boldsymbol{J}=\sum_{s} e_{s} \int \boldsymbol{v} f_{s} d^{3} \boldsymbol{v}
\end{aligned}
$$

assuming the translational symmetry in the $z$ direction. Under the coordinate transformation of $\left(\boldsymbol{x}_{\perp}, \boldsymbol{v}_{\perp}, v_{z}\right) \rightarrow$ 
$\left(-\boldsymbol{x}_{\perp},-\boldsymbol{v}_{\perp}, v_{z}\right)$, we find $\left(\boldsymbol{B}_{\perp}, B_{z}, \boldsymbol{E}_{\perp}, E_{z}, \boldsymbol{J}_{\perp}, J_{z}, \nabla_{\perp}, \partial_{v_{\perp}}, \partial_{v_{z}}\right)$ $\rightarrow\left(-\boldsymbol{B}_{\perp}, B_{z},-\boldsymbol{E}_{\perp}, E_{z},-\boldsymbol{J}_{\perp}, J_{z},-\nabla_{\perp},-\partial_{v_{\perp}}, \partial_{v_{z}}\right)$. As described in Sec. 2.2, we divide $f_{s}, \boldsymbol{B}$, and $\boldsymbol{E}$ into even and odd parity components and rewrite Eq. (30) as follows:

$$
\begin{aligned}
\partial_{t} f_{s+} & +\boldsymbol{v}_{\perp} \cdot \nabla_{\perp} f_{s+}+\frac{e_{s}}{m_{s}}\left(\boldsymbol{E}_{\perp+} \cdot \partial_{\boldsymbol{v}_{\perp}} f_{s-}+\boldsymbol{E}_{z+} \cdot \partial_{\boldsymbol{v}_{z}} f_{s+}\right. \\
& \left.+\boldsymbol{E}_{\perp-} \cdot \partial_{\boldsymbol{v}_{\perp}} f_{s+}+\boldsymbol{E}_{z-} \cdot \partial_{\boldsymbol{v}_{z}} f_{s-}\right) \\
& +\frac{e_{s}}{m_{s}}\left(\left(\boldsymbol{v}_{\perp} \times \boldsymbol{B}_{+}\right) \cdot \partial_{\boldsymbol{v}_{\perp}} f_{s+}+\left(\boldsymbol{v}_{\perp} \times \boldsymbol{B}_{+}\right) \cdot \partial_{\boldsymbol{v}_{z}} f_{s-}\right. \\
& +\left(\boldsymbol{v}_{\perp} \times \boldsymbol{B}_{-}\right) \cdot \partial_{\boldsymbol{v}_{\perp}} f_{s-}+\left(\boldsymbol{v}_{\perp} \times \boldsymbol{B}_{-}\right) \cdot \partial_{\boldsymbol{v}_{z}} f_{s+} \\
& \left.+\left(\boldsymbol{v}_{z} \times \boldsymbol{B}_{+}\right) \cdot \partial_{\boldsymbol{v}_{\perp}} f_{s-}+\left(\boldsymbol{v}_{z} \times \boldsymbol{B}_{-}\right) \cdot \partial_{\boldsymbol{v}_{\perp}} f_{s+}\right)=0
\end{aligned}
$$

$$
\begin{aligned}
\partial_{t} f_{s-} & +\boldsymbol{v}_{\perp} \cdot \nabla_{\perp} f_{s-}+\frac{e_{s}}{m_{s}}\left(\boldsymbol{E}_{\perp+} \cdot \partial_{\boldsymbol{v}_{\perp}} f_{s+}+\boldsymbol{E}_{z+} \cdot \partial_{\boldsymbol{v}_{z}} f_{s-}\right. \\
& \left.+\boldsymbol{E}_{\perp-} \cdot \partial_{\boldsymbol{v}_{\perp}} f_{s-}+\boldsymbol{E}_{z-} \cdot \partial_{\boldsymbol{v}_{z}} f_{s+}\right) \\
& +\frac{e_{s}}{m_{s}}\left(\left(\boldsymbol{v}_{\perp} \times \boldsymbol{B}_{+}\right) \cdot \partial_{\boldsymbol{v}_{\perp}} f_{s-}+\left(\boldsymbol{v}_{\perp} \times \boldsymbol{B}_{+}\right) \cdot \partial_{\boldsymbol{v}_{z}} f_{s+}\right. \\
& +\left(\boldsymbol{v}_{\perp} \times \boldsymbol{B}_{-}\right) \cdot \partial_{\boldsymbol{v}_{\perp}} f_{s+}+\left(\boldsymbol{v}_{\perp} \times \boldsymbol{B}_{-}\right) \cdot \partial_{\boldsymbol{v}_{z}} f_{s-} \\
& \left.+\left(\boldsymbol{v}_{z} \times \boldsymbol{B}_{+}\right) \cdot \partial_{\boldsymbol{v}_{\perp}} f_{s+}+\left(\boldsymbol{v}_{z} \times \boldsymbol{B}_{-}\right) \cdot \partial_{\boldsymbol{v}_{\perp}} f_{s-}\right)=0 .
\end{aligned}
$$

If $f_{s}$ has the even parity component only, namely, $f_{s_{-}}=0$ with $\boldsymbol{E}_{\perp+}=0, \boldsymbol{E}_{z^{-}}=0, \boldsymbol{B}_{\perp+}=0$ and $\boldsymbol{B}_{z-}=0$, Eqs. (34) and (35) lead to

$$
\begin{aligned}
\partial_{t} f_{s+} & +\boldsymbol{v}_{\perp} \cdot \nabla_{\perp} f_{s+}+\frac{e_{s}}{m_{s}}\left(\boldsymbol{E}_{z+} \cdot \partial_{\boldsymbol{v}_{z}} f_{s+}+\boldsymbol{E}_{\perp-} \cdot \partial_{\boldsymbol{v}_{\perp}} f_{s+}\right) \\
\quad+ & \frac{e_{s}}{m_{s}}\left(\left(\boldsymbol{v}_{\perp} \times \boldsymbol{B}_{z+}\right) \cdot \partial_{\boldsymbol{v}_{\perp}} f_{s+}+\left(\boldsymbol{v}_{\perp} \times \boldsymbol{B}_{\perp-}\right) \cdot \partial_{\boldsymbol{v}_{z}} f_{s+}\right. \\
\quad+ & \left.\left(\boldsymbol{v}_{z} \times \boldsymbol{B}_{\perp-}\right) \cdot \partial_{\boldsymbol{v}_{\perp}} f_{s+}\right)=0, \\
\partial_{t} f_{s-} & =0
\end{aligned}
$$

where the coupling of the symmetric part doesn't spontaneously generate $f_{-}$and the distribution function preserves the parity symmetry. If the distribution function has a symmetry for the reversal of the coordinates around the $\mathrm{X}$ point, where $\nabla_{\perp}=0, \boldsymbol{E}_{\perp-}=0$ and $\boldsymbol{B}_{\perp-}=0$ at the X-point, Eq. (36) would be reduced to

$$
\partial_{t} f_{s+}+\frac{e_{s}}{m_{s}}\left(-\partial_{t} A_{z+} \partial_{\boldsymbol{v}_{z}} f_{s+}\right)=-\frac{e_{s}}{m_{s}}\left(\boldsymbol{v}_{\perp} \times \boldsymbol{B}_{z+}\right) \cdot \partial_{\boldsymbol{v}_{\perp}} f_{s+},
$$

where $E_{z^{+}}=-\partial_{t} A_{z+}$ as $\partial_{z}=0$. The r.h.s in Eq. (38) stands for the gyromotion. In the Vlasov-Maxwell system, it is difficult to find the analytic solution of the distribution function at the $\mathrm{X}$-point unless $f_{s+}$ is gyrotropic $\left(\partial_{v_{\perp}} f_{s+}=0\right)$. Assuming $B_{z} \gg B_{\perp}$ and $f_{s+}=F_{M s}+f_{1 s+}$ where $f_{1 s+}$ is the perturbed distribution function, and the Maxwellian distribution becomes independent of the gyrophase, and r.h.s of Eq. (38) vanishes. Thus, Eq. (38) in the leading order is described as

$$
\partial_{t} f_{1 s+}+\frac{e_{s}}{T_{s}} v_{z} F_{M s} \partial_{t} A_{z+}=0,
$$

which is similar to Eq. (18) in the gyrokinetic Vlasov system.
We note that 2-D parity transformation is described regarding the case with a strong guide field, which is different from a standard parity transformation in the 3-D case. In the 3 -D case $(x, y, z) \rightarrow(-x,-y,-z)$, the magnetic field $(\boldsymbol{B})$ is unchanged, since $\boldsymbol{B}$ is an axial vector that changes direction under the 3-D parity transformation. Conversely, in the 2-D case with $(x, y) \rightarrow(-x,-y)$, the coordinate system remains right-handed, and hence, the 2-D parity transformation is constructed by flipping the sign of the perpendicular magnetic field as discussed above.

\section{Beam Instability}

The beam distribution of $\tilde{f}_{\| e}$ along $B_{0}$ is formed by the parallel electric field during the collisionless magnetic reconnection. In this section, we discuss the stability of the beam electrons by considering the $z$ dependence of the perturbations with the wavenumber $k_{z}$. From the gyrokinetic simulation assuming $F_{M s} \gg \tilde{f}_{\| e}$, the total distribution function at the X-point is represented using the shifted Maxwellian distributions with the beam component, $\tilde{U}_{e} \ll v_{t e}$,

$$
F_{M \| e}+\tilde{f}_{\| e} \simeq \frac{n_{0}}{\sqrt{2 \pi} v_{t e}} \exp \left(-\frac{\left(v_{\|}-\tilde{U}_{e}\right)^{2}}{2 v_{t e}^{2}}\right),
$$

where $\tilde{f}_{\| e}=\tilde{U}_{e} v_{\|} / v_{t e}^{2} F_{M \| e}$ from the solution at the X-point, Eq. (19). According to the gyrokinetic ordering, the parallel drift velocity is $O\left(\epsilon_{g k} v_{t s}\right)$, whereas the number density is $O\left(n_{0}\right)$, leading to the deviation from the stationary Maxwellian distribution of the order of $O\left(\epsilon_{g k} F_{M}\right)$. Apart from this ordering, we investigate the instability of the KAWs using the velocity parameter, $V_{b}$.

For simplicity, we consider the local dispersion relation of a plane wave with the wavenumber of $\boldsymbol{k}=\left(k_{\perp}, k_{z}\right)$, neglecting the spatial variation of the background distribution, $F_{0 e}$, with the beam velocity, $V_{b}$. This is a crude assumption; however, it is as meaningful as the first step of the stability analysis and is necessary for determining if the KAWs can be destabilized during the magnetic reconnection even in the limit of plane waves. Thus, we have a linearized set of gyrokinetic equations:

$$
\begin{aligned}
& -i \omega \bar{f}_{s k}+i k_{z} v_{z} \bar{f}_{s k}-\frac{e_{s}}{m_{s}} J_{0 s k}\left(i k_{z} \bar{\phi}_{\boldsymbol{k}_{\perp}}-\frac{i \omega}{c} \bar{A}_{\| \boldsymbol{k}_{\perp}}\right) \partial_{v} F_{0 s}=0 \\
& \sum_{s} e_{s}\left(\int J_{0 s k} \bar{f}_{s} d v_{\|}-\frac{n_{0} e_{s} \bar{\phi}_{\boldsymbol{k}_{\perp}}}{T_{s}}\left(1-\Gamma_{0 s k_{\perp}}\right)\right)=0 \\
& k_{\perp}^{2} \bar{A}_{\| k_{\perp}}=\mu_{0} \sum_{s} e_{s} \int v_{\|} J_{0 s k} \bar{f}_{s k} d v_{\|} \\
& F_{0 e}=\frac{n_{0}}{\sqrt{2 \pi} v_{t e}} \exp \left(-\frac{\left(v_{\|}-V_{b}\right)^{2}}{2 v_{t e}^{2}}\right), F_{0 i}=F_{M \| i}
\end{aligned}
$$

where ${ }^{-}$denotes the linear perturbations. Equations (41) (43) yield the dispersion relation for $T_{i}=T_{e}$ 


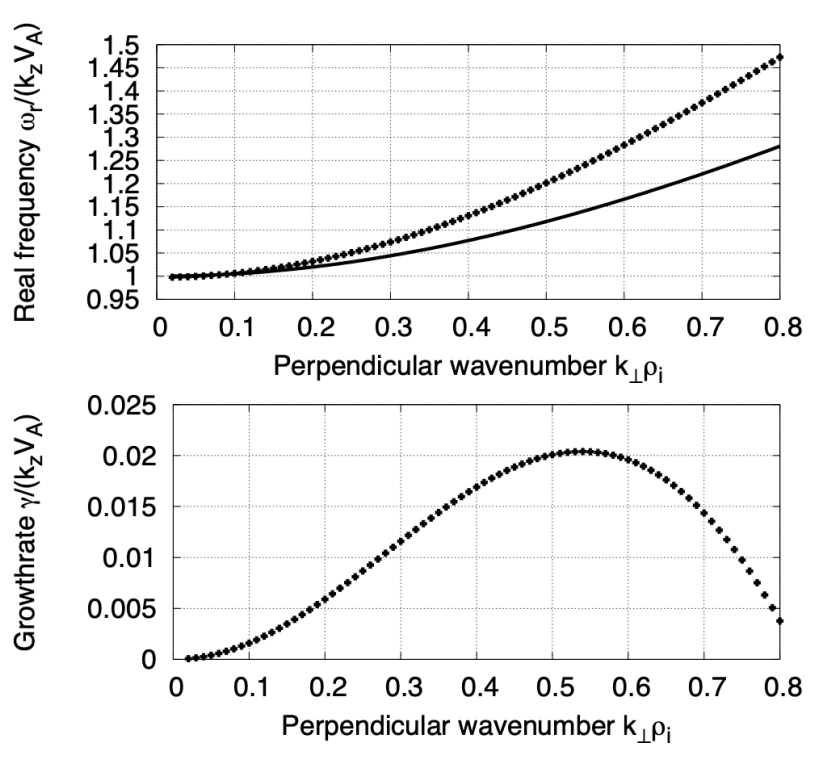

Fig. $1 k_{\perp} \rho_{i}$ vs the real frequency $\omega_{r} / k_{z} V_{A}$ (top panel) and the growth rate $\gamma / k_{z} V_{A}$ (bottom) for $V_{b} / V_{A}=1.5$. The dotted line denotes the numerical solutions of Eq. (45) and the solid line denotes the approximated dispersion relation of the KAWs [3].

$$
\begin{aligned}
& \sum_{s}\left(1-\Gamma_{0 s k}\right)=\left(\left(\frac{\omega}{k_{z} V_{A}}\right)^{2} \sum_{s} \frac{1}{k_{\perp}^{2} \rho_{i}^{2}}\left(1-\Gamma_{0 s k}\right)-1\right) \\
& \quad \times\left(\sum_{s} \Gamma_{0 s k}\left(1+\zeta_{s} Z\left(\zeta_{s}\right)\right)\right),
\end{aligned}
$$

where $\zeta_{e}=\left(\omega-k_{z} V_{b}\right) / k_{z} v_{t e}, \zeta_{i}=\omega / k_{z} v_{t i}$ and $Z\left(\zeta_{s}\right)$ is the plasma dispersion function.

We have conducted a numerical analysis of Eq. (45). The dotted lines in Fig. 1 show the $k_{\perp}$ dependence of the real frequency, $\omega_{r}$, and the linear growth rate, $\gamma$, for $V_{b}=1.5 V_{A}$. The numerical solution of the dispersion relation represents the KAWs, and it is compared with the approximated dispersion relation of the KAWs, $\left(\omega / k_{z} V_{A}=\sqrt{1+k_{\perp}^{2} \rho_{i}^{2}}\right)$ [22] (the solid line in Fig. 1). In the bottom panel, we find the positive growth rate for $0<k_{\perp} \rho_{i}<0.8$, of which the maximum value is 0.02 $k_{z} V_{A}$ around $k_{\perp} \rho_{i} \simeq 0.54$, suggesting the beam-excited KAWs with $\omega / k_{z}=1.25 V_{A}$, which is slightly slower than $V_{b}=1.5 V_{A}$.

Figure 2 shows the maximum growth rates, $\gamma_{\max }$, as functions of the electron beam velocity, $V_{b}$, with different mass ratios of $m_{e} / m_{i}=1 / 100,1 / 200$, and $1 / 400$. Evidently, $\gamma_{\max }$ has positive values when $V_{b}>V_{A}$ for all cases. Namely, if the beam velocity exceeds the Alfvén speed, the KAWs become unstable. We have also observed the dependence of the growth rate on the mass ratio through $V_{A} / v_{t e}$. The critical beam speed of $V_{b}=V_{A}$ normalized by the electron thermal speed, $v_{t e}$, is given by $V_{A} / v_{t e}=\sqrt{m_{e} / m_{i} \beta_{i}}$ for $T_{i}=T_{e}$; it decreases with the increase in the ion-toelectron mass ratio and is less than unity for $\beta_{e}>m_{e} / m_{i}$. Thus, the stability criteria for the KAWs can be lower

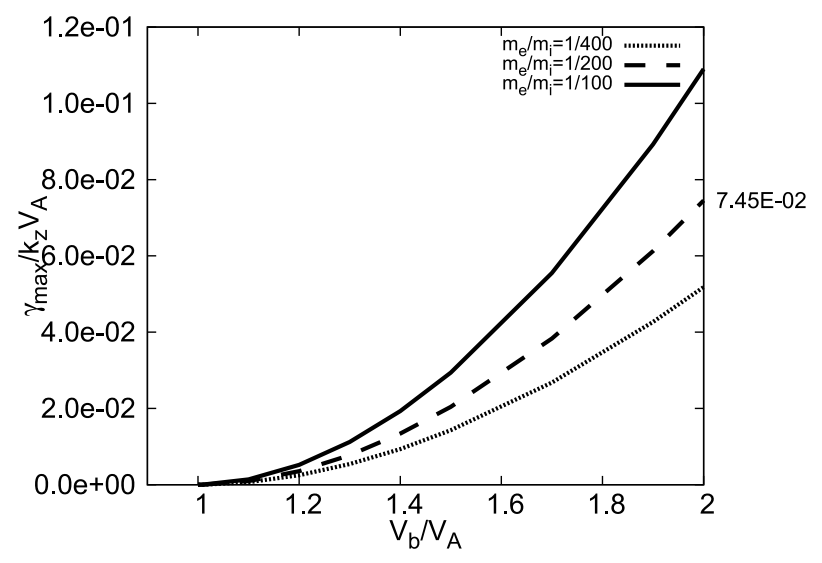

Fig. 2 The maximum growth rate $\gamma_{\max } / k_{z} V_{A}$ plotted for the beam velocity $V_{b} / V_{A}$ for $m_{e} / m_{i}=1 / 100,1 / 200$, and $1 / 400$. In all cases, the KAWs are unstable when the beam velocity exceeds the Alfvén speed.

than that for the Buneman instability (i.e., $V_{b}>v_{t e}$ ). If the normalization is $k_{z} L \rightarrow k_{z}$, the growth rate of the simulation timescale would be $\gamma L / v_{t i}=\gamma L / \sqrt{\beta_{i}} V_{A}$. For example, for $V_{b}=1.5 V_{A}$, the maximum growth rate is $\gamma_{\max } \sim 0.02 V_{A} / L=0.14 v_{t i} / L$.

\section{Simulation Result}

The numerical simulation of the collisionless reconnection is conducted by solving the gyrokinetic equations, Eqs. (1) - (3), to verify the parity symmetry and the structures of the distribution function discussed in Sec. 2.2 and 2.3 and to evaluate the time-development of the electron beam velocity at the X-point. The simulation code uses the Fourier spectral method for the discretization of the real space and for the computation of spatial derivatives. The fourth-order Runge-Kutta-Gill method is applied for the time integration. The used parameters are ratios of mass, temperature, and charge: $m_{e} / m_{i}=1 / 200, T_{e} / T_{i}=1$, $e_{e} / e_{i}=-1, \beta_{i}=0.02$. The normalized skin depth is $d_{e} / \rho_{i}=\sqrt{m_{e} / \beta_{i} m_{i}}=1 / 2$ and the normalized Alfvén speed is $V_{A} / v_{t e}=\sqrt{m_{e} / \beta_{i} m_{i}}=1 / 2$. We set $\epsilon=5 \times 10^{-5}$ and $\left(\tilde{U}_{0 e}, \tilde{U}_{0 i}\right)=\left(-0.31104 v_{t e}, 0\right)$. The simulation box is given by $L_{x} / \rho_{i}=2.5 \pi$ and $L_{y} / \rho_{i}=5 \pi$ in the configuration space and $-5 \leq v_{\|} / v_{t s} \leq 5$ in the velocity space, and it is discretized by $512 \times 512 \times 127$ grid points. The characteristic time scale $L / v_{t i}$ is rewritten by use of the Alfvén time for the reconnecting field, $L_{x} / V_{a \perp}$, as

$$
\frac{L}{v_{t i}}=\frac{\rho_{i}}{L_{x} \sqrt{\beta_{i}}} \frac{L_{x}}{V_{a \perp}} \sim 5.66 \frac{L_{x}}{V_{a \perp}},
$$

where $V_{a \perp}=B_{\perp} / \sqrt{\mu_{0} n_{0} m_{i}}$. The initial condition of $\tilde{f}_{\| s}$ is given by Eq. (8), providing the initial perturbed vector potential written as $\left\langle\tilde{A}_{\|}\right\rangle_{s}(\boldsymbol{x}, t=0)=\tilde{A}_{\|_{0}} \cos \left(2 \pi x / L_{x}\right)$, to produce the sheared magnetic field through the parallel current.

Figure 3 shows snapshots of the magnetic field lines 

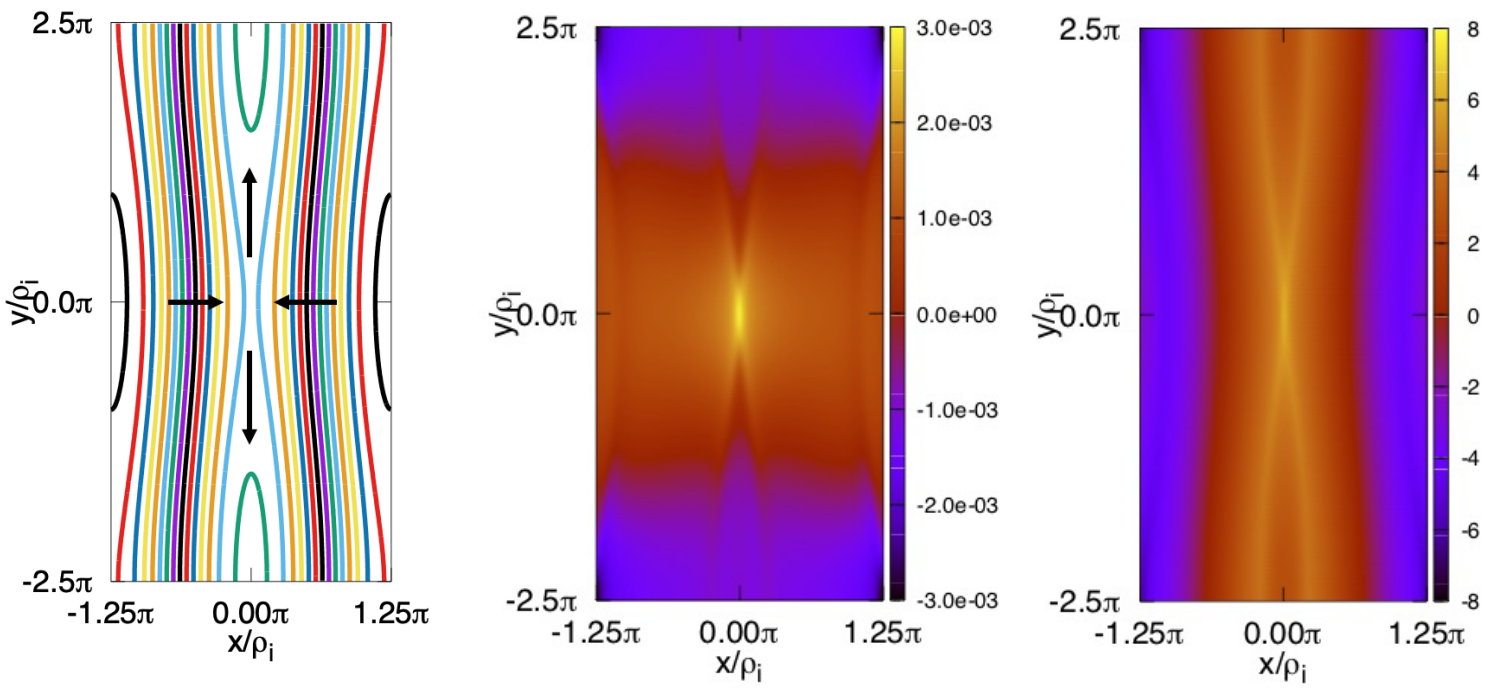

Fig. 3 Structures of (a) the magnetic field lines $\tilde{A}_{\|}$, (b) parallel electric field $\left\langle\tilde{E}_{\|}\right\rangle_{e}$, and (c) parallel current density $\tilde{J}_{\|}$structures on the reconnection plane at $t v_{t i} / L=90$ in the nonlinear phase. Black arrows in Fig. 3 (a) show the direction of the $E \times B$ flow.

(contour of $\tilde{A}_{\|}$), the parallel electric field for electrons $\left\langle\tilde{E}_{\|}\right\rangle_{e}$, and the current density $\left(\tilde{J}_{\|}\right)$during the magnetic reconnection (at $\left.t v_{t i} / L=90\right)$ which takes place on the $\mathrm{X}$ point at $\left(x / \rho_{i}, y / \rho_{i}\right)=(0,0)$. The inflow is parallel to the $x$ - axis and the outflow is parallel to the $y$ - axis. The magnetic reconnection also occurs at the corners because of the doubly periodic boundary condition in $x$ and $y$. The magnetic reconnection at the corner should be the same as that at $x=y=0$, except for the direction of the reconnecting magnetic field because of the symmetry. The reconnection at $x=y=0$ may interact with the reconnection at the corners. We find that the separatrix field lines have an $\mathrm{X}$-shaped structure in the nonlinear phase when the fast reconnection proceeds rapidly. The $\mathrm{X}$-shaped structure along the separatrix is also formed in the profiles of the current density and the electric field, as shown in Fig. 3 (b) and (c) where the rotational symmetry property around the $z$ axis with the angle of $\pm \pi$ with respect to the reconnection point is well preserved. These typical structures have also been reported in previous studies on collisionless reconnection with the two-fluid effect [4].

In the case with a weak or moderately intense guide field, the asymmetry of the diffusion region appears because of the Hall effect [10]. However, in the present case with a strong guide field, the parallel component of the perturbed magnetic field is negligible with respect to the guide field. Thus, the symmetry of the flux function both in the $x$ and $y$ directions is preserved, consistent with the simulation results.

Figure 4 shows the time evolution of the reconnection electric field at the X-point, i.e., $\left\langle\tilde{E}_{\|}\right\rangle_{e}=-\partial_{t}\left\langle\tilde{A}_{\|}\right\rangle_{e}$. The parallel electric field grows exponentially in time in the early phase and the reconnection rate $\left(=\log \left(\left\langle\tilde{E}_{\|}\right\rangle_{e}(t) /\left\langle\tilde{E}_{\|}\right\rangle_{e}(t=\right.\right.$ $0))$ ) is about $0.17 v_{t i} / L$ in the linear growth phase. The

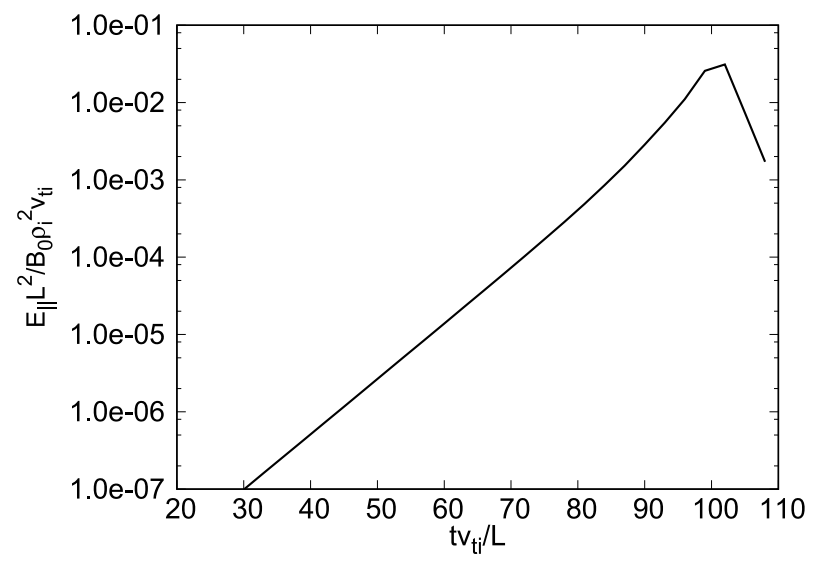

Fig. 4 Time evolution of the reconnection electric field $\left(-\partial_{t}\left\langle\tilde{A}_{\|}\right\rangle_{e}\right)$ at the X-point. The reconnection rate grows rapidly from approximately $t v_{t i} / L=80$, and its peak is at $t v_{t i} / L=100$.

growth rate of $\tilde{E}_{\| e}$ increases nonlinearly after $t v_{t i} / L=80$, indicating the acceleration of the magnetic reconnection and $\tilde{E}_{\| e}$ peaks at $t v_{t i} / L \sim 100$ when the magnetic field lines have almost reconnected. It has been pointed out that the acceleration of the reconnection rate does not occur in the case of the collisional reconnection where the Ohmic dissipation is dominant [23].

Here, we discuss the structures of the electron distribution function observed during the reconnection. Figure 5 shows the spatial structures of the electron distribution function $\tilde{f}_{\| e}$ at $v_{\|} / v_{t e}= \pm 1.03$, plotted on the reconnection $(x-y)$ plane in the nonlinear phase $\left(t v_{t i} / L=90\right)$. At the X-point, $\tilde{f}_{\| l}$ is negative for $v_{\|}>0$ but positive for $v_{\|}<0$ since the electrons are accelerated by the positive electric filed. The perturbed electron distribution function 

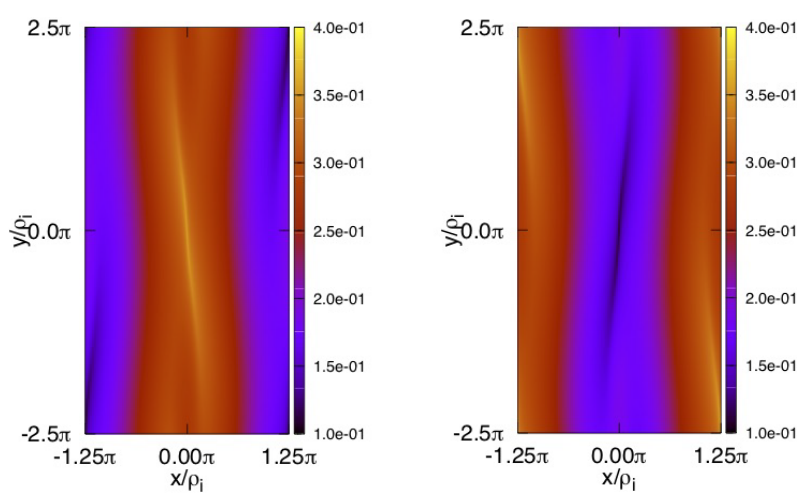

Fig. 5 Electron distribution function on the reconnection plane at $v_{\|} / v_{t e}=-1.03$ (left panel) and $v_{\|} / v_{t e}=+1.03$ (right panel) in the nonlinear phase $\left(t v_{t i} / L=90\right)$.

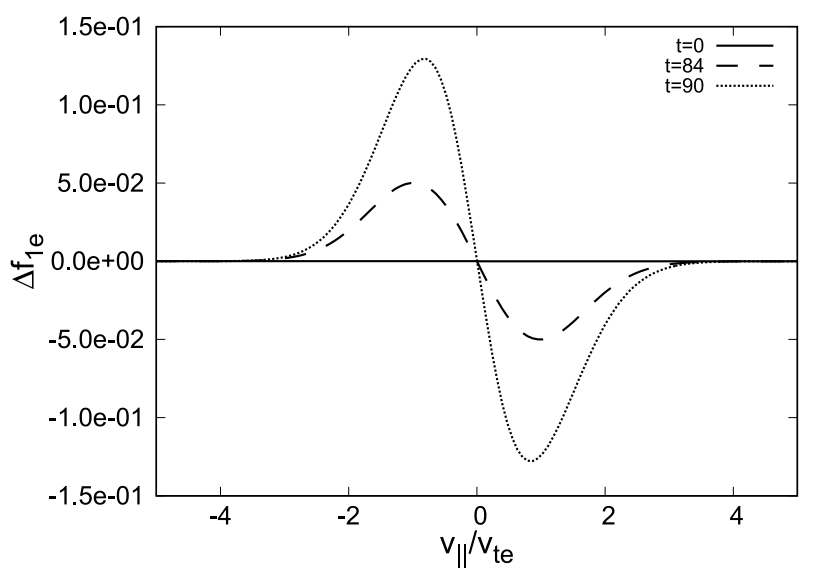

Fig. 6 Velocity-space profiles of the electron velocity distribution function at the X-point for the linear phase $\left(t v_{t i} / L=\right.$ $0)$, and the nonlinear phase $\left(t v_{t i} / L=84,90\right)$.

is also advected along the reconnected field lines, where $\tilde{f}_{\| e}$ in $v_{\|}>0$ is elongated on the different sides of the $x$ and $y$ space from those in $v_{\|}<0$. This is because the distribution function has the anti-symmetry configuration with respect to the coordinate transformation of $\left(x, y, v_{\|}\right) \rightarrow\left(-x, y,-v_{\|}\right)$ and $\left(x, y, v_{\|}\right) \rightarrow\left(x,-y,-v_{\|}\right)[24]$. These structures also have the parity symmetry around the $\mathrm{X}$-point, as given in Eq. (15). The present simulation confirms that the two kinds of symmetry of $\tilde{f}_{\| s}$ are stably preserved during the reconnection process. The velocity space integration of $\tilde{f}_{\| e}$, thus, results in the cross-shaped profile of the current density around the X-point, as shown in Fig. 3 (c).

The deviation of the electron velocity distribution function from the initial condition at the X-point, $\Delta \tilde{f}_{e}(t)=$ $\tilde{f}_{\| e}(t)-\tilde{f}_{\| e}(0)$, is plotted in Fig. 6. Noteworthily, $\Delta \tilde{f}_{e}$ is proportional to $v_{\|} F_{M \| e}$, and agrees with the one derived in Eq. (19). Furthermore, we find that the electron acceleration is enhanced in the nonlinear stage (from $t v_{t i} / L=80$ to 100) as the reconnection electric field increases. The time history of the deviation of the averaged electron ve-

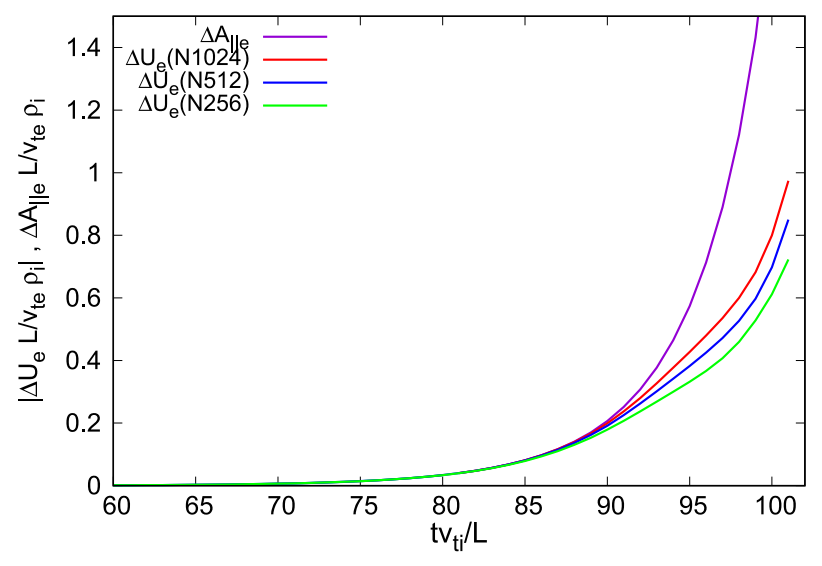

Fig. 7 The deviation of the averaged electron velocity from the initial condition, $\Delta \tilde{U}_{e}$, for $N=256$ (green), 512 (blue) and 1024 (red) at the X-point, reconnected flux $\left(\Delta \tilde{A}_{\|}\right)$ (purple), and Alfvén speed $\left(V_{A}\right)$ (black). The reconnected flux is plotted only for $N=1024$ since the behavior is the same for all cases.

locity from the initial condition at the X-point, $\Delta \tilde{U}_{e}$ given by Eq. (21), and the reconnected magnetic flux multiplied by $e_{e} / M_{e}, \Delta \tilde{A}_{\| e}$ given by Eq. (20), is shown in Fig. 7 where the evolution of the beam velocity for the three cases with different grid numbers of $N \equiv N_{x}=N_{y}=256$ (green), 512 (blue) and 1024 (red) is plotted. According to Eq. (21), the electron acceleration at the X-point should balance with the reconnected flux. Here, the balance of $\Delta \tilde{U}_{e}$ and $\Delta \tilde{A}_{\| e}$ is well satisfied up to $t v_{t i} / L \sim 90$, whereas the difference between the two appears in the nonlinear phase after $t v_{t i} / L \sim 90$, because of the limitation of the numerical resolution. A high beam velocity is found for finer resolutions and is closer to the analytical solution (purple), whereas the reconnected flux is the same for all cases. The peak velocities in each resolution are $\Delta \tilde{U}_{e}(N=256)=0.7228 \epsilon_{g k} v_{t e}$, $\Delta \tilde{U}_{e}(N=512)=0.8498 \epsilon_{g k} v_{t e}$, and $\Delta \tilde{U}_{e}(N=1024)=$ $0.9740 \epsilon_{g k} v_{t e}$.

The subtle improvement in $\Delta \tilde{U}_{e}$ for increasing the numerical resolution is attributed to the sharp structure of the distribution function around the $\mathrm{X}$-point. Figure 8 shows the snapshots of the cross-section of $\Delta \tilde{f}_{e}$ for $N=256,512$ and 1024 at $y=0$ and $t v_{t i} / L=101$. We find that the sharp peak of $\Delta \tilde{f}_{e}$ develops at the X-point, of which the amplitude grows with $N$, whereas the global profile of $\Delta \tilde{f}_{e}$, as well as the current density profile, is well converged. Thus, it is difficult to completely resolve the cusp-shaped structure of $\Delta \tilde{f}_{e}$ with the numerical resolution available, since the cusp width is thin for a high peak value (or $\Delta \tilde{U}_{e}$ ) at the $\mathrm{X}$-point to keep the current density constant. Nevertheless, through the convergence check of $\Delta \tilde{U}_{e}$, we can conclude that the essence of the acceleration process of electrons in the collisionless reconnection is well captured by the present simulations. As shown in Ref. [20], in this simulation, microstructures in the velocity space are not dominant in the profiles of the distribution function. At the 


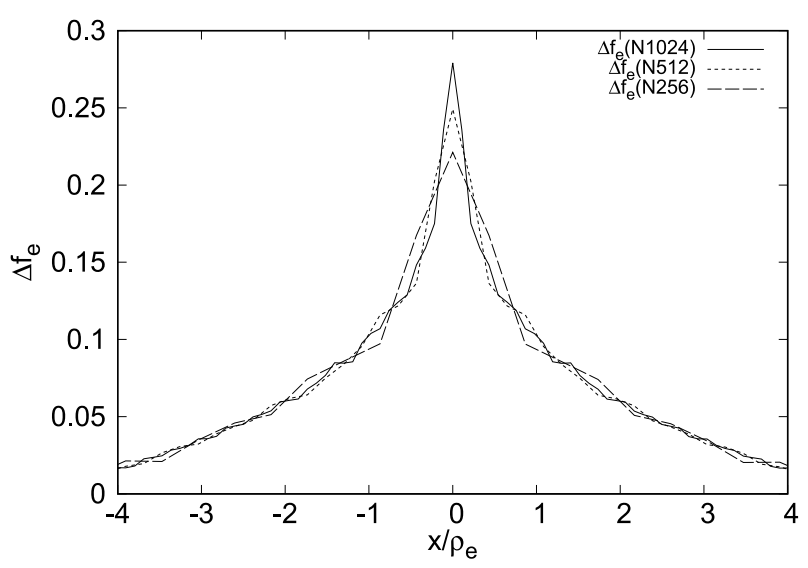

Fig. 8 The cross-section of the deviation of the electron distribution function from the initial condition around the $\mathrm{X}$ point at $t v_{t i} / L=101, \Delta \tilde{f}_{e}$, at $y=0$ for $N=256$ (dashed line), 512 (dotted line), and 1024 (solid line).

$\mathrm{X}$-point where the fine structure develops in the real space, the distribution function in the velocity space is described by the shifted Maxwellian distribution. The numerical errors, thus, mainly stem from the lack of the spatial resolution.

Additionally, we consider the recurrence time $\left(T_{\text {rec }}\right)$ because of the parallel motion of electrons along the perturbed magnetic field. The recurrence time is estimated as $T_{r e c}=2 \pi / k_{\|} \Delta v_{\|}$with $k_{\|}=\left|k_{y} \partial_{x} A_{\|} / B_{0}\right|$ and $\Delta v_{\|}=L_{v} / n_{v}$, where $k_{\|}, \Delta v_{\|}, n_{v}$, and $L_{v}$ are the parallel wavenumber, grid spacing, grid number, and box size in the parallel velocity space, respectively. For $\partial_{x} A_{\|} / B_{0} \sim 0.11 \sin \left(2 \pi x / L_{x}\right)$ estimated from the initial condition, and for the wavenumber $k_{y} \sim 0.4 / \rho_{i}$ of a box size perturbation, we can estimate $T_{r e c}$ as $T_{r e c} \sim 40 \pi L / v_{t i}$ which is comparable with the total simulation time and longer than the reconnection time scale. Furthermore, near the X-point, $T_{\text {rec }}$ becomes longer than the above estimate because of the relatively weak perturbed field. Therefore, the perturbed distribution function is well resolved in the parallel velocity space, and the numerical error related to the velocity space resolution is considered negligible.

In Sec. 4, we demonstrated that the electron beam $\tilde{U}_{e} \sim \epsilon_{g k} v_{t e}$ is generated at the X-point. Combining with the results in Sec. 3, we discuss whether the KAW instability criterion by the electron beam, $\tilde{U}_{e}>V_{A}$ is satisfied through magnetic reconnection. By rewriting the Alfvén speed as $V_{A}=\epsilon_{a} v_{t e}$, the instability condition becomes $1 \gg \epsilon_{g k}>\epsilon_{a}$. In the present simulation, we used $m_{i}=200 m_{e}$, and thus, $\epsilon_{a}=0.5$, since it was numerically difficult to conduct a simulation using the real ionto-electron mass ratio. However, the value of $\epsilon_{a}$ can be reduced smaller when we use realistic parameters, e.g., $\epsilon_{a}=0.07$ for $m_{e} / m_{i}=1 / 1836$ and $\beta_{i}=0.1$. Therefore, there would be a regime satisfying the KAWs instability condition $1 \gg \epsilon_{g k}>\epsilon_{a}$. Additionally, the growth rate of the instability of KAWs, $\gamma_{D R}$, is $\gamma_{D R}=0.0745 V_{A} / L$ for $\tilde{V}_{b}=2 V_{A}=v_{t e}$ and $m_{e} / m_{i}=1 / 200$, as shown in Fig. 2. The growth rate of the simulation timescale is $\gamma_{D R}=0.0745 v_{t i} /\left(\sqrt{\beta_{i}} L\right)=0.526 v_{t i} / L$ which is higher than the linear growth rate of the reconnection, i.e., $0.17 v_{t i} / L$.

The non-dimensional factor, $\epsilon_{a}$, is merely one of the additional parameters, and it can be as small as $\epsilon_{a} \sim \epsilon_{g k}$ so that the beam instability is excited by the accelerated electrons during the collisionless magnetic reconnection while keeping maintaining consistency with the low-speed parallel motion under the gyrokinetic ordering.

If one uses the full-f gyrokinetic equations, which involve the total distribution functions, it is expected that the velocity of the electron beam can be increased with no limitation of $\epsilon_{a} \sim \epsilon_{g k}$. However, the physical mechanism of the full-f model is the same as that discussed in the present study. Namely, the parameter regime discussed above is required only for the delta-f gyrokinetic formulation, which is also relevant to the initial acceleration process in the fullf gyrokinetic reconnection.

Notably, full-f gyrokinetic Vlasov equation obtained by adding the parallel nonlinear term to Eq. (4) can be written as

$$
\partial_{t} F_{t o t s}+\left[\left\langle\phi-p_{\|} A_{\|}\right\rangle_{s}, F_{t o t s}\right]=0
$$

If one uses the canonical momentum $p_{\|}$, i.e., $p_{\|}=$ $v_{\|}+e_{s}\left\langle A_{\|}\right\rangle_{s} / m_{s}$, instead of $v_{\|}$in the full-f gyrokinetic Vlasov equation, dropping the second order term [18]. Equation (46) states that the total distribution function $F_{\text {tots }}\left(x, p_{\|}\right)$at the $\mathrm{X}$-point remains unchanged because of the symmetry constraint. As the reconnection proceeds, thus, $F_{\text {tots }}\left(x, v_{\|}\right)$is shifted in $v_{\|}$according to the change in $\left\langle A_{\|}\right\rangle_{s}$. This means that the parallel acceleration of electrons discussed above can also be found in the full-f approach, where the shifted Maxwellian distribution of the total distribution function, i.e., $F_{\text {tots }}\left(x, v_{\|}\right) \propto \exp \left(-\left(v_{\|}-V_{b}\right)^{2} / 2\right)$, should be found at the reconnection point if we start from the initial Maxwellian distribution in $p_{\|}$.

\section{Summary and Discussion}

We have investigated the phase space structures of the distribution function in collisionless magnetic reconnection with a strong guide field using the gyrokinetic model. The electric field induced by the electron inertia accelerates the electrons at the X-point. From the stability analysis using the dispersion relation, it is suggested that the KAWs can be excited when the electron beam velocity exceeds the Alfvén speed.

Through the discussion on the symmetry of the gyrokinetic equations around the $\mathrm{X}$-point, it is found out that the shifted Maxwellian distribution is formed at the $\mathrm{X}$ point by the reconnection electric field, and the electron beam velocity is proportional to the reconnected flux. This analytic solution is found in the gyrokinetic system but not in the Vlasov-Maxwell system unless the gyrotropic distri- 
bution function is assumed.

From the dispersion relation of a plane wave in the case with the beam electrons, we found that the KAWs are unstable when the beam velocity exceeds the Alfvén speed $\left(\tilde{U}_{e}>V_{A}\right)$. This is also possible from the viewpoint of energy conversion from the magnetic energy to the beam energy. The instability threshold is lower than that of the Buneman instability (i.e. $\tilde{U}_{e}>v_{t e}$ ), if the plasma beta is higher than the electron-to-ion mass ratio.

We also performed gyrokinetic simulations to investigate the structures of the electron distribution function during the collisionless magnetic reconnection in the strong guide field. The fine structure of the electron distribution function is formed along the separatrix field lines on the reconnection plane. The fine structures preserve the symmetry even in the nonlinear evolution. Thus, at the X-point, the electron velocity distribution function develops in proportion to the shifted Maxwellian distribution $\left(v_{\|} F_{M \|}\right)$ as predicted by the theoretical analysis on symmetry. The observed beam velocity is in good agreement with the estimate given by the reconnected flux, provided the fine structures are well resolved. A possible scenario for the generation of the anomalous resistivity is, thus, deduced as follows. In the collisionless magnetic reconnection, the electric field induced at the X-point accelerates electrons. Namely, the electron inertia sustains the reconnection electric field. This results in the formation of an electron beam along the guide field; moreover, the KAWs are destabilized when the beam velocity exceeds the Alfvén speed. Notably, the electron thermal velocity is higher than the Alfvén speed, when the electron beta is larger than the electron-to-ion mass ratio. This implies that the KAW can be more easily destabilized than the Buneman instability in space and fusion relevant plasmas with the guide field.

In the present stability analysis of KAWs, we have considered a plane wave in Eqs. (41)-(43), where the background profile, such as the spatial inhomogeneity of $\tilde{A}_{\|}$, is not included. It is necessary to investigate the stability of the beam electrons with the background inhomogeneity of the total distribution function and the reconnection magnetic field: this investigation is in progress and will be reported elsewhere.

\section{Acknowledgments}

This work is partly supported by JSPS KAKENHI Grant number, JP16H04086. Computations were per- formed on the "Plasma Simulator" (FUJITSU FX100) of National Institute of Fusion Science (NIFS) with the support and under auspices of the NIFS Collaboration Research program (NIFS18KNWT001).

[1] D. Biskamp, Magnetic Reconnection in Plasmas (Cambridge Univ., Cambridge, 2000) p.49.

[2] E. Priest, Magnetic Reconnection MHD Theory and Applications (Cambridge Univ., Cambridge, 2009) C.5.

[3] R.C. Davidson, N.T. Gladd, C.S. Wu and J.D. Huda, Phys. Fluids 20, 301 (1977).

[4] E. Cafaro, D. Grasso, F. Pegoraro, F. Porcelli and A. Saluzzi, Phys. Rev. Lett. 80, 20 (1998).

[5] M. Otaviani and F. Porcelli, Phys. Rev. Lett. 71, 23 (1993).

[6] M. Hesse, K. Schindler, J. Birn and M. Kuznetsova, Phys. Plasmas 6, 1781 (1999).

[7] J. Birn, J.F. Drake, M.A. Shay, B.N. Rogars, R.E. Denton, M. Hesse, M. Kuznetsova, Z.W. Ma, A. Bhattacharjee, A. Otto and P.L. Pritchett, J. Geophys. Res. 106, 3715 (2001).

[8] B.N. Rogers, R.E. Denton, J.F. Drake and M.A. Shay, Phys. Rev. Lett. 87, 195004 (2001).

[9] T.A. Carter, H. Ji, F. Trinchouk, M. Yamada and R.M. Kulsrud, Phys. Rev. Lett. 88, 015001 (2001).

[10] J.P. Eastwood, M.A. Shay, T.D. Phan et al., Phys. Rev. Lett. 104, 205001 (2010).

[11] Y.-H. Liu, W. Daughton, H. Li and S.P. Gary, Phys. Plasmas 21, 022113 (2014).

[12] M. Hirota, Y. Hattori and P.J. Morrison, Phys. Plasmas 22, 052114 (2015).

[13] R. Horiuchi and T. Sato, Phys. Plasmas 6, 12 (1999).

[14] T. Moritaka, R. Horiuchi and H. Otani, Phys. Plasmas 14, 102109 (2007).

[15] R.B. Torbert, J.L. Burch, B.L. Giles, D. Gershman, C.J. Pollock, J. Dorelli, L. Avanov, M.R. Argall, J. Shuster, R.J. Strangeway, C.T. Russel et al., Geophys. Res. Lett. 43, 5918 (2016).

[16] H. Che, Phys. Plasmas 24, 082115 (2017).

[17] T.S. Hahm, W.W. Lee and A. Brizard, Phys. Fluids 31, 1940 (1988).

[18] X. Garbet, Y. Idomura, L. Villard and T.H. Watanabe, Nucl. Fusion 50, 043002 (2010).

[19] M.J. Pueschel, F. Jenko, D. Told et al., Phys. Plasmas 18, 112102 (2011).

[20] A. Ishizawa and T.-H. Watanabe, Phys. Plasmas 20, 102116 (2013).

[21] R. Numata and N.F. Loureiro, Phys. Plasmas 81, 305810201 (2015).

[22] A. Hasegawa, J. Geophys. Res. 81, 5093 (1976).

[23] M. Hirota, J. Plasma Fusion. Res. 92, 12 (2016).

[24] A. Ishizawa, S. Maeyama, T.-H. Watanabe and H. Sugama, J. Plasma Phys. 81, 43510203 (2015). 